\section{Adjuvant GnRH agonists do not increase cardiovascular mortality}

Risk factors for cardiovascular disease, including impaired insulin sensitivity, increased fat mass and high levels of cholesterol and triglycerides, are exacerbated by gonadotropinreleasing hormone $(\mathrm{GnRH})$ agonists. Analysis of data from the phase III Radiation Therapy Oncology Group trial 85-31 has shown that this elevation of risk does not translate into a greater likelihood of death due to cardiovascular disease in men with locally advance prostate cancer.

Men ( $n=945$, median age 70 years) were randomized to either radiotherapy alone or to radiotherapy plus adjuvant androgen deprivation therapy with the $\mathrm{GnRH}$ agonist goserelin (Zoladex ${ }^{\circledR}$, AstraZeneca, Wilmington, DE). During a median follow-up period of 8.1 years, 574 patients died; 117 of these deaths were related to cardiovascular disease.

There was no significant association between treatment with goserelin and cardiovascular mortality, defined as sudden death or death from myocardial infarction, cardiac arrest, cardiovascular arrhythmia, cardiomyopathy, coronary artery disease, cardiovascular disease or congestive heart failure. Re-analysis using different definitions of cardiovascular mortality (i.e. on the basis of age, prevalent cardiovascular disease or diabetes) and adjustment for missing data and censoring of data from patients treated with salvage goserelin, also failed to detect an association.

Long-term adjuvant treatment with $\mathrm{GnRH}$ agonists does not increase cardiovascular mortality. The authors warn, however, that these drugs might still contribute to noncancer deaths via a noncardiovascular mechanism.

Original article Efstathiou JA et al. (2008) Cardiovascular mortality after androgen deprivation therapy for locally advanced prostate cancer: RTOG 85-31. J Clin Oncol 27: 92-99

\section{Gastric bypass surgery improves sexual function in morbidly obese men}

In obese men, sexual dysfunction is associated with hypertension, diabetes, and metabolic syndrome. Weight loss after gastric bypass surgery should, therefore, improve sexual function.
Dallal et al. used the Brief Male Sexual Function Inventory, which evaluates erectile function, ejaculation, sexual drive, satisfaction and sexual problems, to assess 97 morbidly obese men (mean BMI $51 \mathrm{~kg} / \mathrm{m}^{2}$, mean age 48 years) before and after gastric bypass surgery. Of these patients, $51 \%$ had diabetes and $70 \%$ had hypertension requiring treatment prior to the operation. Compared with published, referenced controls, preoperative sexual function was poor in the study cohort; it improved notably after surgery decreased mean BMI to $31.8 \mathrm{~kg} / \mathrm{m}^{2}$. After an average follow-up period of 19 months, only $21 \%$ and $40 \%$ of patients required treatment for diabetes and hypertension, respectively. These factors were not, however, associated with change in sexual function. Rather, improvements in sexual function were positively correlated with the increasing amount of weight lost.

The finding that sexual dysfunction can be resolved after substantial weight loss should be a strong motivator to obese men considering a healthier lifestyle, the authors conclude.

Original article Dallal RM et al. (2008) Sexual dysfunction is common in the morbidly obese male and improves after gastric bypass surgery. J Am Coll Surg 207: 859-864

\section{High-risk prostate cancer is prevalent among low-income men}

With the advent of PSA screening, the proportion of men with low-risk, organ-confined prostate cancer at diagnosis has greatly increased in recent years. However, an analysis by researchers in California suggests that this trend does not extend to low-income, uninsured men.

Miller et al. conducted a retrospective analysis of 570 patients enrolled during 2001-2006 in the IMPACT (Improving Access, Counseling and Treatment for Californians with Prostate Cancer) program, a state-run initiative aimed at providing prostate cancer care to low-income Californians. Participants had no medical insurance and a maximum household income of twice the US Federal Poverty Level. At diagnosis, $51 \%$ of participants had a PSA level $>10 \mathrm{ng} / \mathrm{ml}, 50 \%$ had a Gleason score $\geq 7,43 \%$ had disease stage T2 or greater, and 19\% had metastatic cancer. Of the participants enrolled in $2006,45 \%$ had high-risk prostate cancer at 